\title{
¿Puntos de partida y llegada simétricos? Una tipología basada en la relación educación-empleo
}

\author{
Jorge-Alberto Arellano-Martínez e Iván-Alejandro Salas-Durazo
}

\section{RESUMEN}

En el presente escrito se propone una tipología para clasificar y explicar la relación educación superior-empleo a partir de la conjunción de elementos asociables a la sociología de la juventud, la teoría del capital humano y la política de educación superior. Se empleó la metodología de análisis cualitativo comparado mediante el uso de lógica booleana para el análisis multidimensional. Se analizaron estudiantes de la carrera de Abogado de la Universidad de Guadalajara al ingreso y próximos al egreso. Como principal resultado se evidenció la heterogeneidad de estudiantes con respecto al empleo destacando su carácter dinámico durante el tránsito escolar.

Palabras clave: educación y empleo, trayectoria escolar, estudiantes que trabajan, taxonomía, modelos de análisis, México. 
Pontos de partida e chegada simétricos? Uma tipologia baseada na relação educação-emprego

\section{RESUMO}

No presente escrito se propõe uma tipologia para classificar e explicar a relação educação superior - emprego a partir da conexão de elementos associáveis à sociologia da juventude, a teoria do capital humano e a política de educação superior. Se utilizou a metodologia de análise qualitativo - comparado mediante o uso de lógica booleana para a análise multidimensional. Se analisaram estudantes do curso de direito da Universidad de Guadalajara à entrada e próximos à conclusão do curso. Como principal resultado se evidenciou a heterogeneidade de estudantes com respeito ao emprego destacando seu caráter dinâmico durante o trânsito escolar.

Palavras chave: educação e emprego, trajetória escolar, estudantes que trabalham, taxonomia, modelos de análises, México.

\section{Symmetrical Departure and Arrival Points? A Typology Based on the Education-Employment Relationship}

\section{ABSTRACT}

This paper proposes a typology to classify and explain the relationship between higher education and employment based on the conjunction of elements associated with the sociology of youth, the theory of human capital and the higher education policies. The methodology of comparative qualitative analysis was used using Boolean logic for multidimensional analysis. The authors compared the cases of students just enrolled at the Law School of the University of Guadalajara and students close to graduating. The main result was the heterogeneity of students with regard to employment, highlighting its dynamic character during school trajectory.

Key words: education and employment, school trajectory, working students, taxonomy, analysis models, Mexico. 


\section{Introducción}

La expansión del subsistema de educación superior suscitado en México durante finales de la década de los ochenta y principios de los noventa se caracterizó por el establecimiento de programas y acciones diseñadas mayormente desde la perspectiva institucional. Durante más de 25 años de maduración de la política educativa se han fortalecido ejes estratégicos asociados a la evaluación de la calidad, ampliación de la oferta educativa, desarrollo de infraestructura, creación de nuevas modalidades, vinculación con sectores sociales, empresariales y gubernamentales, consolidación del posgrado y responsabilidad financiera, entre otros (Comas, Buendía, Fresán y Gómez, 2014; Buendía, 2007; Rubio, 2006). Asimismo, se han incorporado ejes transversales asociables al desarrollo sustentable, internacionalización, equidad, responsabilidad social y transparencia (González, Meira y Martínez, 2015; Alcántara y Navarrete, 2014; Valarezo y Túñez, 2014; Wit, 2011; González, Wagenaar y Beneitone, 2004).

Independientemente del desarrollo de los mecanismos de intervención - gubernamental e institucional- la orientación de las instituciones de educación superior (IES) poco ha cambiado. A lo largo de los últimos 25 años, la política educativa de nivel superior se ha caracterizado en un primer momento por acciones encaminadas al aumento de la cobertura y en una segunda instancia a elevar la calidad educativa como resultado de la expansión sin regulación. Buendía et al. (2017) señalan que los programas implementados se focalizan en dos grandes aspectos: 1) aumentar el nivel de habilitación de la planta académica, y 2) la actualización de los planes de estudio y los métodos de enseñanza. En ese sentido los estudiantes, en su carácter de piezas clave en el sistema, han sido históricamente conceptualizados de manera ambigua y hasta cierto punto reduccionista. Como señala Guzmán (2017) la concepción institucional del estudiante ideal está rebasada ante la realidad que enfrentan actualmente los universitarios. Desde su perspectiva no existe un único tipo de estudiante sino que por el contrario está presente una amplia gama de conglomerados diferenciados por sus objetivos personales y los mecanismos de mediación de los diversos ámbitos de la vida con la educación superior. El análisis de las características de los estudiantes universitarios se ha distinguido por estudiar la superposición del tránsito escolar con aspectos tales como el empleo y la etapa vital de la juventud (De Garay, Miller y Montoya, 2016; Salas y Flores, 2016a; Cuevas y De Ibarrola, 2015). En cualquier caso, implícitamente se busca entender a los sujetos desde una perspectiva compleja y no sólo como población objetivo del sistema educativo.

El objetivo del presente escrito consistió en la construcción de una tipología de estudiantes universitarios orientada a profundizar en el entendimiento de la relación educación-empleo considerando elementos asociables a la sociología de la juventud, a la teoría del capital humano y a la política educativa de educación superior. Como principal aportación se aplica la lógica booleana para categorizar comportamientos complejos a partir de la relación entre variables en el marco de la metodología del Análisis Cualitativo Comparado. Para evidenciar su capacidad explicativa se analizaron los estudiantes de la carrera de Abogado de las modalidades escolarizada y semiescolarizada durante el segundo semestre de 2016 considerando dos grupos: a) alumnos de primer ingreso y b) próximos al egreso. De esta manera se pudieron abordar de manera indirecta los cambios en las trayectorias laborales de los universitarios.

\section{Una aproximación para entender la naturaleza compleja del estudiante universitario}

En México el estudio de las trayectorias universitarias se ha caracterizado por su abordaje desde diversos enfoques teóricos y metodológicos. Las representaciones sociales, identidades, experiencias 
y expectativas académicas y laborales han sido el foco de atención de una serie de trabajos que buscan conocer el rol del universitario en los últimos años (Guzmán, 2017). Estudios previos se han concentrado en identificar y clasificar el espectro de perfiles que convergen en las instituciones educativas (Cortez, García y Altamirano, 2015; Cuevas, 2007; Ramírez, 2013; Weiss, 2012; De Garay, 2003). Sin embargo, este tipo de estudios son insuficientes tomando en cuenta el papel central que juegan los estudiantes en la dinámica universitaria.

Del mismo modo, a pesar de que se reconoce que los estudiantes cuentan con historias particulares y una vida social compleja, parece existir un consenso implícito que los define como jóvenes (Miller, 2009; De Garay, 2003). Lo anterior repercute en las políticas educativas ya que al hacer referencia a la cobertura neta es importante mencionar que se toma en cuenta únicamente a la población dentro de la cohorte de 19 a 23 años. Por tanto, las acciones gubernamentales implícitamente han considerado como población objetivo natural a los jóvenes. En ese sentido, los programas públicos parecen visualizar al estudiante universitario como joven con ciertas características estáticas que han prevalecido pese a los cambios dinámicos en aspectos políticos, económicos y sociales. Complementariamente, Trow (1974) matiza lo anterior al señalar que la educación superior de un país se encuentra en la fase de educación de élite cuando la matrícula no supera el 15\% de la tasa neta de cobertura. Por su parte, una tasa de cobertura nacional entre el 15\% y el 50\% ubicaría al país en una fase de educación de masas. Por último, puede considerarse en la fase de universalización cuando la educación superior está por encima del $50 \%$ de la cobertura neta. De manera que durante los últimos 25 años la política educativa ha transitado de una población atendida elitista a una masificada sin que ello hubiere significado necesariamente una transición en la orientación o en diversificación escalonada de la atención.
$\mathrm{Al}$ analizar al estudiante universitario y su grado de madurez es importante tomar en cuenta que el estudio de la juventud desde la sociología cuenta con diversos enfoques. Por esta razón, los criterios para delimitar el concepto aún no son concluyentes. Resulta pertinente aclarar que existen diferentes perspectivas para abordar el concepto juventud (Singly, 2005). Desde una visión empirista se visualiza al joven como un ser dentro de periodos de vida estandarizados y homogéneos, los cuales avanzan en ciclos vitales. La crítica a este enfoque argumenta que la agrupación de sujetos que sólo tienen en común el periodo en que nacieron no justifica que se aborde a la juventud como un constructo social homogéneo, ya que no existe un orden social universal basado en la segmentación de rangos de edades (Brunet y Pizzi, 2013). Igualmente existe un enfoque que señala a la juventud como una etapa de la biografía de cada individuo que abarca desde el inicio de la pubertad física hasta la emancipación familiar plena. Es decir, desde el momento en que una persona puede reproducirse hasta que adquiere la capacidad de autonomía mediante el término de los estudios, el inicio de una actividad laboral y el inicio de una familia propia (Mora y De Oliveira, 2009; Casal, García, Merino y Quesada, 2006). En ese sentido, el alargamiento de la escolarización, la precarización del empleo y la postergación del matrimonio han provocado una flexibilización de la juventud como categoría social (Brito, 1998; Flaquer, 1997).

Por otra vía paralela, el estudio de la inclusión de los universitarios a la vida laboral ha aumentado en las últimas décadas (Salas y Flores, 2016a; Carillo y Ríos, 2013; Salas y Murillo, 2013; De Garay 2009; Callender, 2008; Casal et al., 2006; Guzmán, 2004; De Garay y Casillas, 2002). Por tanto, visualizar al estudiante ideal universitario como aquel que se dedica de manera exclusiva a su formación académica podría potenciar el riesgo de omitir al sector de estudiantes universitarios que por necesidad o voluntad han decidido incorporarse al mercado laboral antes de finalizar sus estudios de educación superior. Por 
tanto, se considera pertinente comprender al universitario como un ser complejo y multidimensional diferenciado por sus trayectorias laborales, intereses, prioridades y ocupaciones (Guzmán, 2017; De Garay y Casillas, 2002). Factores como la necesidad económica, la consecución de mayor experiencia profesional o la búsqueda de independencia han impulsado un aumento en madurez del universitario, visto desde el enfoque biográfico de la sociología de la juventud. Al mismo tiempo que los jóvenes universitarios buscan alcanzar la maduración durante su estancia en las IES, existe un grupo de personas que al momento de iniciar sus estudios universitarios ya han alcanzado la emancipación familiar y la integración al mercado laboral, es decir, elementos asociables a la madurez social (Miller, 2009).

Aunado a lo anterior, la teoría de la elección racional en conjunto con la teoría del capital humano complementan la explicación de la complejidad del estudiante universitario al señalar que los individuos con mayor preparación académica y mayor experiencia tienden a obtener ingresos más altos. De manera que la educación superior es una inversión al mediano y largo plazo que se superpone con otros aspectos asociables a los ciclos vitales (Barceinas, Oliver, Raymond y Roig, 2001).

Dependiendo del grado de desarrollo y los intereses de cada individuo, la educación superior puede ser vista desde tres posturas: 1) como un bien de consumo que no modifica la productividad; 2) como un filtro que diferencia a los individuos sin afectar la productividad, o 3) como una inversión al capital humano el cual vuelve más productivo al individuo y genera mejores salarios (Johnson, 1975). En ese sentido, el trabajar durante los estudios, la experiencia laboral, el tipo de contratación, la afinidad y orientación de la formación con el empleo repercuten en los itinerarios de los estudiantes no sólo al egreso sino también durante su tránsito escolar.

$\mathrm{Al}$ apoyarnos del concepto del capital humano, la educación superior se concibe como una inversión la cual es constantemente analizada por los estudiantes para establecer su permanencia en el subsistema de educación superior al balancear el mayor salario que esperan recibir con la inversión de recursos y tiempo que demanda su preparación académica (Mincer, 1974; Becker, 1964). A raíz de esto, la experiencia y la inclusión temprana a la vida laboral para algunos universitarios puede resultar una estrategia eficiente para optimizar la inversión al permitir diferenciarse de su pares en un mundo cada vez más competitivo (Guzmán, 2017; Salas y Murillo, 2013; De Garay, 2009; Guzmán, 2004). Esto cobra particular sentido en países como México que cuentan con índices de desempleo relativamente bajos pero con una generalizada precarización laboral (De Oliveira, 2006; Hualde y Serrano, 2005). Al respecto, Guadarrama, Hualde y López (2016) señalan que la precariedad se caracteriza por incertidumbre en el horizonte temporal dada la alta probabilidad de perder el empleo. Por su parte, Reynoso (2017) la dimensiona como la disminución de la protección de los derechos laborales desde un punto de vista jurídico. Macías (2013) profundiza lo anterior al atribuir la precarización laboral a los esquemas de contratación actuales derivados de las reformas a la Ley Federal del Trabajo.

\section{Metodología y descripción de la muestra}

El Análisis Cualitativo Comparado (QCA por sus siglas en inglés) surge como una alternativa a los métodos tradicionales a través de la mediación de elementos cuantitativos y cualitativos. Salas y Flores (2016b) señalan que su aplicación surge como una respuesta para el estudio de la realidad social considerando que implícitamente este tipo de fenómenos parten de supuestos de multidimensionalidad, indeterminación y ambigüedad en términos conceptuales y operativos. En esencia, esta aproximación busca explicar un fenómeno mediante la combinación de variables, aspectos o elementos a través de la lógica booleana mediante tablas de verdad (Yamasaki y Spreitzer, 2006). En otras 
palabras, las diferentes combinaciones son definidas a priori, anticipando un valor resultante de salida para cada enunciado. Este proceso de definición de variables y comportamientos se denomina conocimiento sustantivo y es el resultado de la integración de teorías, conceptos, tendencias, experiencias previas y el testimonio de expertos.

Para esta aplicación se diseñaron tablas de verdad en dos niveles de análisis. La primera con relación a elementos asociables a tres aspectos identificados en la literatura que forman parte del estudio de las trayectorias laborales de los universitarios: la sociología de la juventud, la teoría del capital humano y los supuestos implícitos de la política educativa de nivel superior. El segundo nivel se refirió a la combinación de las salidas individuales como insumos para una nueva tabla de verdad en la que se definió una tipología compleja sobre la relación de educación superior-empleo desde la perspectiva de los estudiantes.

En lo que respecta a los elementos asociables a la sociología de la juventud se destaca la edad como una de las variables principales para explicar los ciclos vitales. Asimismo el estado civil, particularmente la vida en pareja, permite identificar el proceso de transición de la juventud a la vida adulta. Con base en cifras del INEGI correspondientes a 2016, el cambio entre un grupo mayoritario soltero a uno casado o en unión libre se encuentra a los 30 años de edad, por lo que se utilizó este valor como criterio para establecer el límite para diferenciar a dos grupos de estudiantes.

No obstante, la propia sociología de la juventud refiere que la juventud no es per se un estado claramente definido que se alcanza y supera en momentos determinados de la vida. Por el contrario, tiene que ser entendida como una condición social compleja la cual está integrada por una serie de elementos psicosociales que van más allá de la edad (Brito, 1998). Casal et al. (2006) profundizan al referir que la juventud se entiende como un proceso que busca concretar la emancipación familiar y profesional a través de la búsqueda de independencia de la familia de origen para obtener un mayor grado de autonomía en la toma de decisiones. Esto tiene como consecuencia un cambio en el rol que juegan los individuos en términos de dependencia y atribuciones. Con base en lo anterior, las variables propuestas para la construcción de este eje analítico están conformadas por: edad, estado civil, composición familiar y el destino principal de los ingresos. En la tabla 1 se muestran dichas variables y los criterios de clasificación empleados para la valoración.

Por consiguiente y para efectos del presente escrito la combinación de variables conlleva a una salida etiquetada lingǘsticamente definida en el grupo de "joven" o en el de "adulto". Para ejemplificar, en un extremo se podría encontrar un estudiante menor a 30 años, soltero, que vive con sus padres y que los ingresos que obtiene son destinados exclusivamente a sus necesidades, el cual estaría dentro de la clasificación de "joven". En el otro extremo, un estudiante clasificado como "adulto" sería mayor a 30 años, vive en pareja, en una composición familiar diferente a la de origen y con el destino de sus ingresos hacia la manutención de su familia. Sin embargo, la definición de todas las posibles combinaciones supone en algunos casos ambigüedad puesto que potencialmente se cuenta con cierto grado de pertenencia a ambos grupos. Para resolver lo anterior, mediante el $Q C A$ y particularmente con la lógica booleana es posible construir una tabla de verdad que refleje y categorice dichas combinaciones, inclusive si empíricamente alguna de ellas no está presente. Cabe aclarar que la variable de destino del ingreso involucra la totalidad de los recursos utilizados por los estudiantes independientemente de su fuente. Es decir, considera tanto el salario como apoyos familiares, institucionales o de cualquier otro tipo dependiendo de las características de cada encuestado. En el Anexo 1 se muestra la tabla de verdad asociada a este eje analítico.

Por su parte, la teoría del capital humano ha sido empleada para cuantificar las diferencias salariales entre grupos de trabajadores a partir de su grado 
Tabla 1. Variables y criterios de clasificación empleados para el eje analítico asociado a la sociología de la juventud

\begin{tabular}{|c|c|c|}
\hline Variable & Etiquetas lingüísticas para la clasificación & Criterios / valores \\
\hline \multirow{2}{*}{ Edad } & Joven & Menor a 30 años \\
\hline & Adulto & Igual o mayor a 30 años \\
\hline \multirow{5}{*}{ Estado civil } & Soltero & Soltero \\
\hline & \multirow{4}{*}{ Actualmente o alguna vez en pareja } & Casado \\
\hline & & Unión libre \\
\hline & & Divorciado \\
\hline & & Viudo \\
\hline \multirow{5}{*}{ Composición familiar } & Núcleo de origen & Con los padres \\
\hline & \multirow{4}{*}{ Independiente al núcleo de origen } & Independiente \\
\hline & & En pareja \\
\hline & & Con amigos \\
\hline & & Otros \\
\hline \multirow{2}{*}{ Destino del ingreso } & Personal & Exclusivamente al sujeto \\
\hline & De apoyo a terceros & Cubrir gastos a terceras personas \\
\hline
\end{tabular}

Fuente: elaboración propia.

de habilitación y su capacidad productiva (Salas y Murillo, 2013). En esencia, Becker (1964) señala que la productividad de los trabajadores puede ser explicada por la combinación de la formación general y específica. En otras palabras, es posible relacionar el nivel de habilitación tanto con la formación académica como con la experiencia profesional.

Por lo anterior, se propusieron cinco categorías para estimar el grado de habilitación de los estudiantes universitarios. En principio, se diferencian los grupos de estudiantes que se dedican exclusivamente a sus estudios de aquellos que están insertados en el mercado laboral. Una segunda categoría busca develar la experiencia profesional de los estudiantes haciendo una aproximación a la formación específica. Lo anterior podría clarificar o cuestionar uno de los principios más contundentes que supone la teoría del capital humano y que define que los individuos con mayor nivel educativo y mayor experiencia tienden a obtener mayores salarios (Barceinas, Oliver, Raymond y Roig, 2001). Una tercera categoría aborda el tipo de contratación debido a que se considera que el tipo de contrato puede afectar la permanencia del trabajador y por ende la estabilidad y seguridad en el desarrollo de capital humano del individuo (Anaya, 2009; Loyzaga, 2009). Las categorías de afinidad carrera-empleo y orientación de la formación, parten de las conclusiones del trabajo de Salas y Flores (2016b) los cuales encuentran que los estudiantes que laboran en ocupaciones relacionadas con su carrera pueden desarrollar mayor experiencia y madurez en la vida laboral, a diferencia de trabajos sin mucha relación. En la tabla 2 se muestran los criterios de clasificación de las variables empleadas para la construcción del eje asociado a la teoría del capital humano. De manera similar al eje anterior, la construcción de las tablas de verdad que definen las salidas se muestran en el Anexo 2. 
Para el eje asociado a la política educativa se establecen tres categorías: la dedicación al estudio, edad y los apoyos económicos que reciben los estudiantes. Cabe destacar que sobre el último elemento se consideran dos niveles: el primero asociado a la esfera gubernamental en apoyos tales como las Becas de Manutención - antes PRONABES -; mientras que el segundo se refiere a las instituciones educativas a través de sus programas de becas, internados, ayudantías, entre otros.

La dedicación académica busca dilucidar el nivel de prioridad que guarda la educación con respecto a la vida laboral en términos del tiempo dedicado a cada una. Para ello, se emplea como referencia lo que para la Organización Internacional del Trabajo (OIT) es una jornada laboral corta, es decir, menor a 20 horas por semana.
En lo que respecta a la edad, se emplea como criterio de diferenciación la cohorte de edad utilizada para calcular la cobertura de educación superior tanto por el Consejo Nacional de Población (CONAPO) como por la Secretaria de Educación Pública (SEP), es decir, de 19 a 24 años. En ese sentido, esta variable posibilita clasificar a los estudiantes a partir de la población objetivo, planteada en la política educativa.

Finalmente, se consideran los apoyos económicos destinados a la manutención de los estudiantes que en esencia tienen como objetivo un mayor aprovechamiento académico. Puede incluir entre otras becas, pasantías, ayudantías de investigación provenientes de la propia institución o de cualquier otra fuente pública o privada. En la tabla 3 se muestra un resumen de las variables empleadas y su clasificación. Asimismo, en el Anexo 3 se muestran las tablas de verdad correspondientes.

Tabla 2. Variables y criterios de clasificación empleados para el eje analítico asociado a la teoría del capital humano

\begin{tabular}{|c|c|c|}
\hline Variable & Etiquetas lingüísticas para la clasificación & Criterios / valores \\
\hline \multirow{2}{*}{ Situación laboral } & Desocupado & No trabaja \\
\hline & Ocupado & Trabaja \\
\hline \multirow{4}{*}{ Experiencia laboral } & Nula & Menor a 1 año de experiencia \\
\hline & Poca experiencia & Entre 1 y 3 años de experiencia \\
\hline & Trayectoria en desarrollo & Entre 3 y 5 años de experiencia \\
\hline & Trayectoria en consolidación & Mayor a 5 años de experiencia \\
\hline \multirow{2}{*}{ Empleo por el tipo de contratación } & Informal & Sin contrato por escrito \\
\hline & Formal & Con contrato por escrito \\
\hline \multirow{2}{*}{ Percepción de afinidad carrera-empleo } & Baja & Percepción de baja afinidad \\
\hline & Alta & Percepción de alta afinidad \\
\hline \multirow{2}{*}{ Percepción de la orientación de la formación } & Al estudio & Percepción de orientación al estudio \\
\hline & Al empleo & Percepción de orientación al empleo \\
\hline
\end{tabular}

Fuente: elaboración propia. 
Tabla 3. Variables y criterios de clasificación empleados para el eje analítico asociado a la política educativa

\begin{tabular}{|l|l|l|}
\hline \multicolumn{1}{|c|}{ Variable } & Etiquetas lingüísticas para la clasificación & \multicolumn{1}{c|}{ Criterios / valores } \\
\hline \multirow{4}{*}{ Dedicación al estudio } & Exclusiva & No trabaja \\
\cline { 2 - 3 } & Orientada al estudio & Jornada laboral menor a 20 horas por semana \\
\cline { 2 - 3 } & Orientada al trabajo & Jornada laboral igual o mayor a 20 horas por semana \\
\hline \multirow{2}{*}{ Edad } & Dentro de la cohorte de cobertura & Menor a 25 años \\
\cline { 2 - 3 } & Fuera de la cohorte de cobertura & Igual o mayor a 25 años \\
\hline \multirow{2}{*}{$\begin{array}{l}\text { Apoyo económico institucional y/o } \\
\text { externo }\end{array}$} & Con apoyo & Beneficiario de apoyos \\
\cline { 2 - 3 } & Sin apoyo & No recibe apoyo \\
\hline
\end{tabular}

Fuente: elaboración propia.

En un sentido integrativo y multidimensional se consideraron las salidas de cada eje analítico como insumos para la consolidación de una tipología compleja que permita categorizar a los estudiantes a partir de la relación educación superior y empleo. En la tabla 4 se muestran las variables y criterios propuestos. Asimismo, en el anexo 4 se muestran las tablas de verdad correspondientes. De tal manera que de acuerdo con la clasificación propuesta existen cuatro posibles tipos: 1) estudiante, el cual se refiere a los sujetos que de manera exclusiva se dedican a sus estudios; 2) estudiante que trabaja, aquel que combina los estudios y el trabajo aunque con mayor énfasis al primero; 3) trabajador que estudia, complementario al tipo anterior aunque con mayor orientación al trabajo y; 4) transición, para aquellos casos que combinan los estudios y el trabajo de manera equilibrada y sin que predomine una en particular.

Cabe destacar que la metodología empleada posibilita crear salidas a partir de la combinación de entradas, por lo que operativamente se consideró para algunas salidas la etiqueta lingüística "transición” en casos en los que existían elementos simultáneamente asociables a los grupos de "estudiantes que trabajan" y "trabajadores que estudian". Esto supone superioridad analítica ya que puede evitarse forzar condiciones que restan capacidad explicativa por la ambigüedad inherente. Mejor aún, esta herramienta hace posible crear grupos entre las fronteras que existen entre rangos haciendo posible la clasificación de la incertidumbre.

Tabla 4. Variables y criterios de clasificación empleados para la tipología de estudiantes con respecto a la relación educación superior-empleo

\begin{tabular}{|c|c|c|}
\hline Teoría o concepto & Clasificación & Etiquetas lingüísticas para la clasificación \\
\hline \multirow{2}{*}{ Sociología de la juventud } & \multirow{2}{*}{ Etapa de la vida } & Joven \\
\hline & & Adulto \\
\hline \multirow{4}{*}{ Teoría del capital humano } & \multirow{4}{*}{ Nivel de habilitación } & Nulo \\
\hline & & Bajo \\
\hline & & Medio \\
\hline & & Alto \\
\hline \multirow{2}{*}{ Política educativa } & \multirow{2}{*}{ Tipo de estudiante } & Estudiante ideal \\
\hline & & Estudiante atípico \\
\hline
\end{tabular}


Para validar la capacidad explicativa de la tipología se encuestó a los estudiantes de primer ingreso y próximos al egreso de la carrera de Abogado que se imparte dentro del Centro Universitario de Ciencias Sociales y Humanidades (CUCSH) de la Universidad de Guadalajara (UDG) durante los meses de octubre y noviembre del 2016. La decisión de analizar este grupo consiste en que dicha licenciatura cuenta con características que permiten encontrar y diferenciar varios conglomerados, destacando los siguientes criterios: 1) la UDG cuenta con la mayor concentración de estudiantes en el estado de Jalisco, tanto en términos agregados como en la carrera analizada; 2) la oferta de esta carrera se realiza en 14 centros universitarios de los cuales el CUCSH atiende a la mayoría; 3) para el caso del centro universitario analizado la carrera se imparte en las modalidades escolarizada y semiescolarizada; 4) la matrícula en ambas modalidades es la mayor del centro universitario por encima de las demás licenciaturas ofertadas; 5) por su nivel de consolidación en el tiempo y características de la planta académica se puede considerar como representativa de la propia UDG, y 6) al considerar primer ingreso y próximo egreso se consideran diferentes etapas de la trayectoria escolar.

Partiendo de las estadísticas institucionales de la UDG (2016) en el ciclo escolar 2016-B fueron admitidos 612 aspirantes a la licenciatura de Derecho en el CUCSH, 412 en la modalidad escolarizada y 200 en la semiescolarizada. Así, para los estudiantes de primer ingreso se encuestaron 80 estudiantes en la modalidad escolarizada y 70 en la semiescolarizada, partiendo de un nivel de confianza del 95\% y un margen de error del 5\%. Para el caso de los alumnos próximos al egreso y considerando el sistema por créditos que opera en la UDG, se realizó una aproximación con el mismo número de muestras considerando la estabilidad en el ingreso a esa carrera en los últimos años.

Un elemento a tomar en cuenta es que la modalidad semiescolarizada cuenta con mayor flexibilidad horaria que la escolarizada. Por tal razón, Gallegos, Gallegos, Vázquez y Sarracino (2017) destacan que existen diferencias significativas entre los estudiantes de ambas modalidades particularmente en términos de la edad.

\section{Análisis}

Para llevar a cabo el análisis se procedió a la clasificación de los datos obtenidos a partir de los ejes planteados en la metodología, considerando tanto el agregado de estudiantes como diferenciados por su etapa en la trayectoria escolar (nuevo ingreso/ próximo al egreso) y la modalidad educativa (escolarizada/semiescolarizada). Lo anterior se llevó a cabo en dos niveles: 1) de manera individual por cada eje considerando la sociología de la juventud, la teoría del capital humano y los supuestos de la política de educación superior, y 2) a partir de la combinación de las salidas de dichos ejes para la construcción de una tipología que explique la relación educación superior-empleo.

Derivado de la clasificación de los estudiantes a partir de los ejes analíticos asociados a la sociología de la juventud, la teoría del capital humano y de la política educativa se identificó la naturaleza compleja e intrincada de los universitarios, ya que simultáneamente se superponen características diferenciadas de acuerdo con la perspectiva teórica o conceptual empleada. En principio, la metodología del QCA permite establecer - y en este caso, clasificar- comportamientos a partir de la combinación de variables. Esto puede ser llevado a cabo en diferentes niveles con la intención de encontrar una explicación más robusta. Para efectos del presente escrito, el análisis se llevó a cabo en dos momentos, uno asociado a cada eje de manera individual y otro basado en la combinación de todos los factores para establecer una tipología basada en la relación educación-empleo. En la figura 1 se muestra la distribución de estudiantes a partir de los ejes analíticos planteados. 
Figura 1. Clasificación de los estudiantes de Abogado del Cusch

\section{a) Eje asociado a la sociología de la juventud}

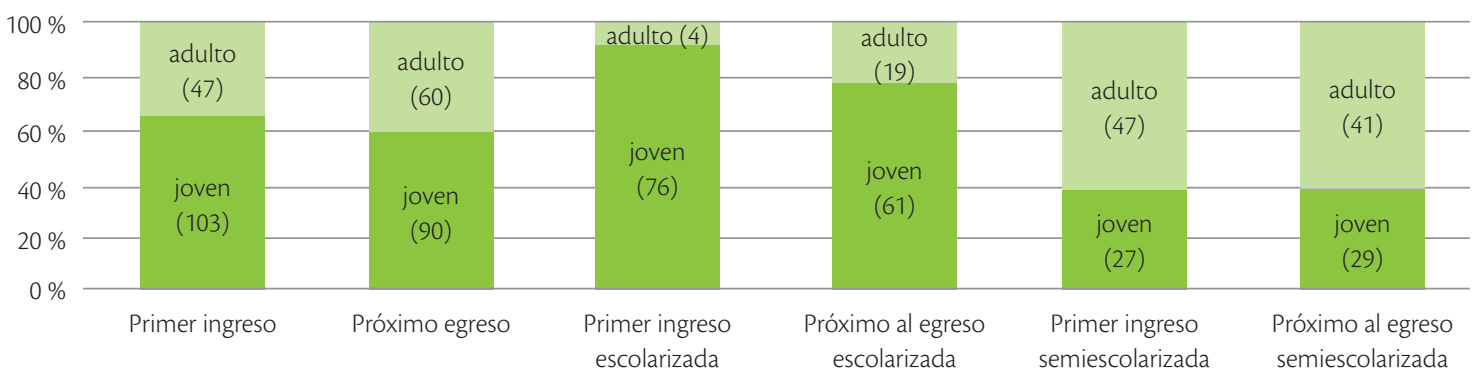

b) Eje asociado a la teoría del capital humano

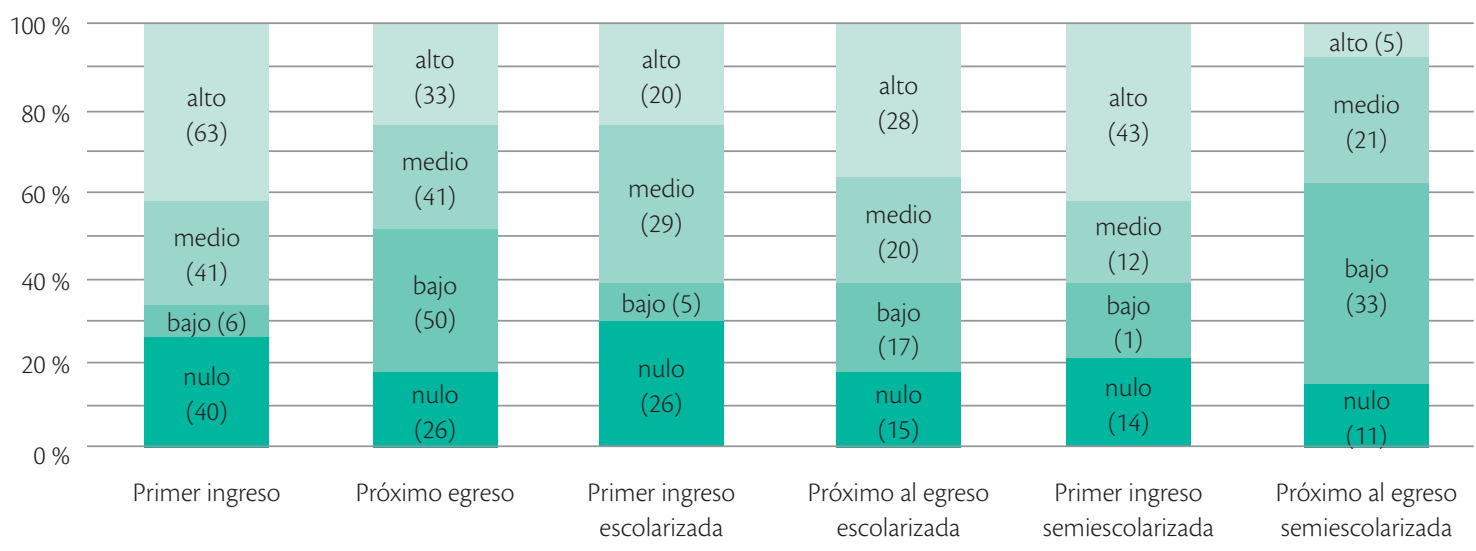

c) Eje asociado a la política educativa

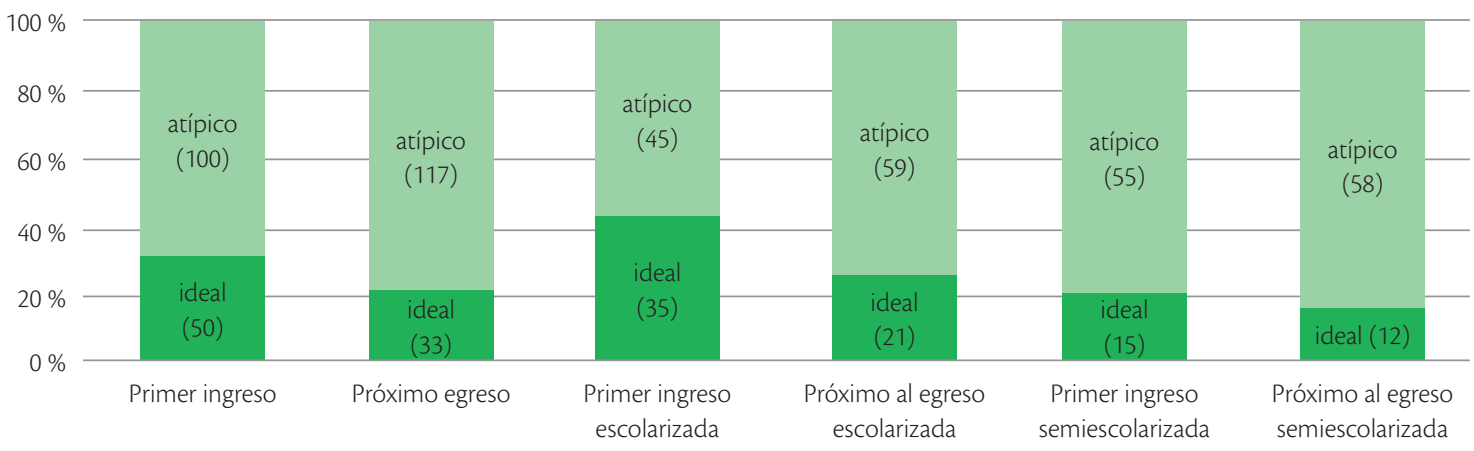


Como se ilustra, los estudiantes universitarios, lejos de ser un grupo homogéneo, presentan características complejas que trascienden a su carácter de estudiantes. En lo que respecta a la sociología de la juventud, se asume que el tránsito por el subsistema de educación superior coincide con la transición de la etapa de joven a adulto. Sin embargo, se presenta evidencia para matizar tal afirmación. Desde esta lógica y a partir de la información recabada, la modalidad educativa es informalmente un espacio donde se diferencian dos grupos de estudiantes. Mientras que en la modalidad escolarizada efectivamente se valida que el grupo mayoritario puede ser clasificado como joven; en la modalidad semiescolarizada convergen sujetos con atributos asociables a la etapa adulta en términos de independencia familiar y económica. Asimismo, no se observan diferencias considerables entre los grupos de nuevo ingreso y próximos al egreso. Esto podría suponer que en términos agregados la transición de la juventud a la adultez en este caso se realiza mayormente en una etapa previa o posterior a los estudios universitarios.

En complemento con lo anterior, los estudiantes universitarios se incorporan informalmente al mercado laboral con diferentes finalidades que van desde la necesidad económica hasta el desarrollo de habilidades y relaciones que impacten positivamente al egreso. En ese sentido, Cuevas y De Ibarrola (2015) destacan que la combinación de educación y empleo potencialmente genera sinergia para el desarrollo de competencias al egreso aunque advierten que lo anterior está condicionado por la congruencia entre la carrera y el empleo ya que de otra forma podría tener efectos contraproducentes. De esta manera, los elementos asociables a la teoría del capital humano nos permiten estimar la etapa de la trayectoria laboral que se superpone a los estudios. En ese sentido, se identifica que de manera consistente existe una mayor habilitación en términos generales de los estudiantes próximos al egreso con respecto de sus contrapartes de primer ingreso. Esto reafirma la idea de que existe una tendencia actual entre los universitarios a combinar los estudios con el trabajo como una regularidad y no como excepción. De igual forma, la modalidad también se identifica como elemento de diferenciación para esta variable encontrando un puente que vincula a la etapa de la vida de la adultez con una mayor consolidación laboral.

Con respecto a los supuestos detrás de la política educativa, los resultados obtenidos hacen evidente la asimetría que existe entre la realidad de los universitarios con respecto a su construcción institucional. Como se muestra, es un porcentaje minoritario el grupo de sujetos que se dedica exclusivamente a los estudios. En ese tenor, conforme los estudiantes avanzan en su trayectoria escolar distan cada vez más del ideal combinándose con los elementos planteados para el eje analítico de la teoría del capital humano.

A partir de lo anterior, cada eje en particular proporciona elementos para validar la idea de que el estudiante universitario es complejo ya que cuenta con matices asociables a diferentes fases de la vida. En un sentido integrador, se construyó una tipología compleja con el objetivo de clasificar a los sujetos desde una perspectiva orientada a la relación que guardan con el empleo. En la figura dos se muestra la distribución de estudiantes. 
Figura 2. Tipología de estudiantes a partir de la relación educación superior-empleo

a) Estudiantes de primer ingreso

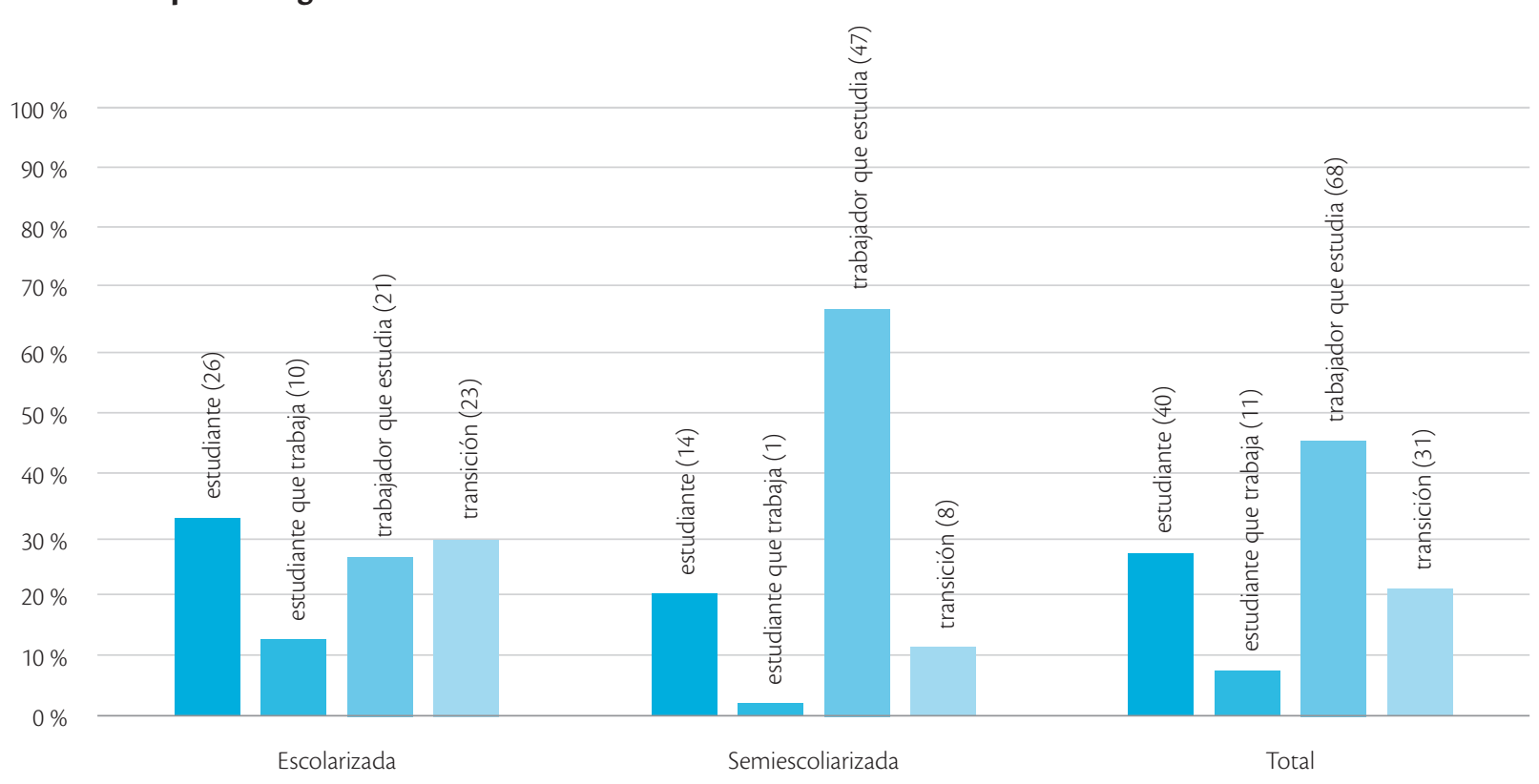

a) Estudiantes próximos al egreso

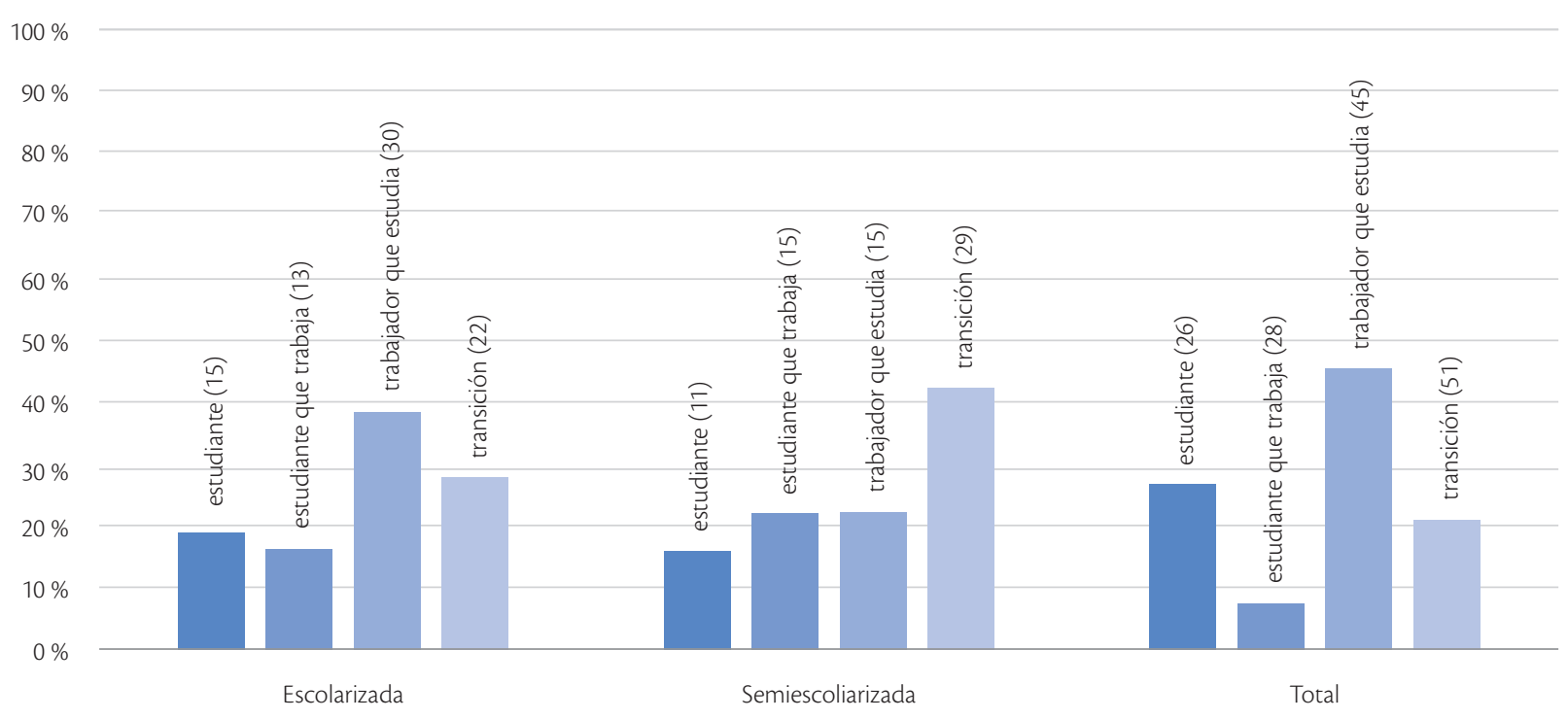


Como ya se anticipaba, el estudiante universitario no puede ser concebido como un tipo único. En este caso la clasificación propuesta evidencia que el estudiante ideal es cada vez menos representativo, inclusive al ingreso. $\mathrm{Al}$ respecto, se destaca que contrario a lo esperado existe un gran número de estudiantes que ingresan a la universidad con un empleo. Esto en principio rompe dos supuestos. El primero, más tradicional, de que el estudio superior y el empleo son secuenciales y mutuamente excluyentes; mientras que el segundo de carácter más emergente, sugiere la combinación del estudio y el trabajo como algo positivo, aunque implícitamente se aborda como el estudiante que se incorpora al trabajo y no tanto como el trabajador que se incorpora a los estudios superiores. En este caso la modalidad educativa fue una variable que diferencia claramente dos grupos de estudiantes.

$\mathrm{Al}$ egreso, el argumento de la incorporación masiva al empleo se vuelve todavía más contundente. Por una parte, se puede identificar que los estudiantes han "adelantado" informalmente sus trayectorias laborales posiblemente con la finalidad de mejorar las condiciones laborales al egreso; aunque simultáneamente, y en particular en el caso de la modalidad escolarizada, cohabita la noción de la prolongación de la juventud en términos de independencia. Estas combinaciones complejas conducen a fortalecer el argumento de Guzmán (2017) al señalar que la conceptualización monolítica del estudiante universitario ya está rebasada.

Por otra parte, la metodología empleada permitió la construcción de un tipo denominado "en transición". Esto resultó particularmente útil en el análisis ya que permitió evitar clasificaciones forzadas en detrimento de la calidad explicativa. Al contrario, hizo evidentes dos aspectos clave para el análisis de las trayectorias laborales: su componente compleja e inherentemente ambigua y su aspecto dinámico ya que posiblemente ese grupo se encuentra en la transición de la juventud a la adultez, el cual lejos de ser dicotómico pareciera ser difuso.

\section{Conclusiones}

En el presente escrito se propone una tipología para entender la manera en la que los estudiantes combinan el empleo con la educación superior desde la conjunción de tres vertientes teórico-conceptuales: la sociología de la juventud, el capital humano y los escenarios esperados en la política educativa de nivel superior. Para ello, se destaca el uso de la metodología de $Q C A$ la cual permitió diferenciar y categorizar a través de la lógica booleana las principales características de cada perspectiva analítica. Dicho en otras palabras, la creación de grupos se basó en la combinación no lineal de factores partiendo del principio de incertidumbre presente en la realidad social. Esto hace posible la identificación de elementos comunes y de diferenciación entre sujetos en términos de la relación educación superior-empleo.

Para ello, se empleó como validación empírica el caso de la carrera de Abogado impartida en la UDG. Como principal aportación se identificó que mediante los criterios propuestos el concepto de "estudiante ideal" presenta matices y variaciones que en principio están condicionados por la etapa de la trayectoria laboral y complementariamente para este caso, por la modalidad educativa. Al ingreso se identificó que en esta etapa de la trayectoria educativa la proporción de estudiantes que trabajan es mucho mayor a la esperada haciendo suponer que la realidad actual de los jóvenes implica un adelanto en términos de las etapas de la vida planteadas en la sociología de la juventud. Esto abre la puerta a estudios que profundicen en conceptualizar la noción de empleo previo a la universidad, el cual prácticamente no ha sido estudiado en el contexto mexicano.

En una etapa próxima al egreso los resultados obtenidos son más parecidos a lo esperado en el sentido de una incorporación masiva al mercado de trabajo aunque con sus diferencias en términos del nivel de habilitación. En ese sentido, los estudiantes clasificados como jóvenes se caracterizan por contar con menor experiencia en promedio que sus contrapartes 
en la etapa adulta. Esto implica diferenciación en cuanto a las trayectorias laborales considerando que en ambos casos están en el paso previo a convertirse en profesionistas.

Por lo anterior es factible afirmar que los puntos de partida y de llegada, es decir al ingreso y al egreso, son asimétricos ya que se identificaron varios grupos que distan de la concepción tradicional del estudiante. Esto tiene particular relevancia al remitirse a los supuestos implícitamente planteados en la política educativa de nivel superior. En un sentido general, el escenario ideal asume al estudio y al trabajo como etapas secuenciales en las que se privilegian incentivos tales como becas para postergar la incorporación laboral a fin de maximizar el aprovechamiento académico. De manera que la única aproximación al mercado de trabajo desde la perspectiva institucional se reduce a la prestación de servicio social y las prácticas profesionales. Asimismo, programas públicos tales como el del Primer Empleo están orientados a personas sin antecedentes laborales ante el IMSS, sin registro como trabajador eventual o en su carácter de trabajador permanente no haber excedido los nueve meses, es decir, que para el caso de los estudiantes universitarios implícitamente se considera que su aproximación al empleo ha sido escasa e informal. Por lo anterior, este trabajo proporciona elementos que sirven como insumos para reorientar algunas de las acciones desarrolladas tanto en la política educativa como en las universidades para transformar el concepto monolítico sobre el estudiante universitario por uno más flexible en el que se reconozcan grupos de estudiantes con características y necesidades diferenciadas.

Finalmente se destaca el uso de la metodología del QCA como una vía para integrar y conceptualizar fenómenos complejos. Esto en algún sentido contribuye a robustecer las explicaciones dado que la realidad social no debería ser reducida a una variable. Es decir, su uso permite operativizar constructos para avanzar en la profundidad del análisis. Por ejemplo, transitar de la concepción de la juventud como sinónimo de edad para conceptualizarla como un estado en el que está en juego el grado de emancipación considerando algunas de sus facetas (edad, vida en pareja, empleo, paternidad). De esta manera es posible clasificar características bajo el respaldo de la integración de teorías o conceptos sin omitir la incertidumbre y ambigüedad inherente al estudio social. 


\section{Referencias}

Alcántara, Armando y Zaira Navarrete (2014), "Inclusión, equidad y cohesión social en las políticas de educación superior en México", en Revista Mexicana de Investigación Educativa, vol. 19, núm. 60, Ciudad de México, Consejo Mexicano de Investigación Educativa, pp. 213-239.

Anaya, Federico (2009), "La estabilidad en el empleo", en Prontuario de Actualización Fiscal, núm. 504, México, Grupo Editorial Gasca, pp. 115-117.

Barceinas, Fernando, Josep Oliver, José Luis Raymond y José Luis Roig (2001), "Hipótesis de señalización frente a capital humano", en Revista de Economía Aplicada, núm. 26, Zaragoza, Facultad de Ciencias Económicas, pp. 125-145.

Becker, Gary (1964), Human Capital, Nueva York, Columbia University Press.

Brito, Roberto (1998), "Hacia una sociología de la juventud. Algunos elementos para la deconstrucción de un nuevo paradigma de la juventud", en Última Década, núm. 9, Valparaíso, Centro de Estudios Sociales, pp. 1-7.

Brunet, Ignasi y Alejandro Pizzi (2013), "La delimitación sociológica de la juventud”, en Última década, vol. 21, núm. 38, Valparaíso, Centro de Estudios Sociales, pp. 11-36.

Buendía, Angélica, Susana García, Rocío Grediaga, Monique Landesman, Roberto Rodríguez, Norma Rondero, Mario Rueda y Héctor Vera (2017), "Queríamos evaluar y terminamos contando: alternativas para la evaluación del trabajo académico", en Perfiles Educativos, núm. 39, vol. 157, pp. 200-220.

Buendía, Angélica (2007), "El concepto de calidad: una construcción en la educación superior", en Reencuentro, núm. 50, México, Universidad Autónoma Metropolitana (UAM)-Unidad Xochimilco, pp. 28-34.

Callender, Claire (2008), "The impact of term-time employment on higher education students' academic attainment and achievement", en Fournal of Education Policy, vol. 23, núm. 4, Londres, Taylor \& Francis Group, pp. 359-377.

Carrillo, Salvador y Jesús Gerardo Ríos (2013), “Trabajo y rendimiento escolar de los estudiantes universitarios.
El caso de la Universidad de Guadalajara, México", en Revista de la Educación Superior, vol. 42, núm. 166, México, Asociación Nacional de Universidades e Instituciones de Educación Superior (ANUIES), pp. 9-34.

Casal, Joaquim, Maribel García, Rafael Merino y Miguel Quesada (2006), "Aportaciones teóricas y metodológicas a la sociología de la juventud desde la perspectiva de la transición”, en Papers. Revista de Sociología, núm. 79, Barcelona, Universidad Autónoma de Barcelona, pp. 21-48.

Comas, Óscar, Angélica Buendía, María Magdalena Fresán e Isabel Gómez (2014), "El PIFI en las universidades públicas: de la decisión racional a la legitimidad institucional", en Revista de la Educación Superior, vol. 43, núm. 169, México, ANUIES, pp. 47-67.

Cortez, Nolvia, Arelys García y Adriana Altamirano (2015), "Estudiantes de retorno en México: estrategias emprendidas para acceder a una educación universitaria", en Revista Mexicana de Investigación Educativa, vol. 20, núm. 67, México, Consejo Mexicano de Investigación Educativa (COMIE), pp. 1187-1208.

Cuevas, José Fernando y María De Ibarrola (2015), "Aprender en la simultaneidad. La perspectiva de los estudiantes que trabajan sobre los saberes y competencias que construyen", en Revista Mexicana de Investigación Educativa, vol. 20, núm. 67, México, COMIE, pp. 1157-1186.

Cuevas, Yazmín (2007), "Representaciones sociales de estudiantes y profesores. La UNAM, el segundo hogar", en Prácticas y representaciones en educación superior, México, IISUE-UNAM/ Plaza y Valdés, pp. 49-84.

De Garay, Adrián, Dinorah Miller e Iván Montoya (2016), "Una misma institución, estudiantes diferentes. Los universitarios de nuevo ingreso de las unidades Azcapotzalco y Cuajimalpa de la UAM", en Sociológica, vol. 31, núm. 88, México, UAM-Azcapotzalco, pp. 95-140.

De Garay, Adrián (2009), ¿Y cuatro años después? De cómo los estudiantes de una generación transitan por la universidad, México, UAM-Azcapotzalco. 
De Garay, Adrián (2003), "Una mirada a los jóvenes universitarios mexicanos", en Nuevas miradas sobre los jóvenes, México/Quebec, Secretaría de Educación Pública/Instituto Mexicano de la Juventud/Office Québec-Amériques pour la Jeunesse/Observatoire Jeunes et Societé, pp. 45-56.

De Garay, Adrián y Miguel Casillas (2002), "Los estudiantes como jóvenes. Una reflexión sociológica", en Fóvenes, culturas e identidades urbanas, México, UAM/ Miguel Ángel Porrúa, pp. 245-262.

De Oliveira, Orlandina (2006), "Jóvenes y precariedad laboral en México", en Papeles de Población, vol. 12, núm. 49, Toluca, Universidad Autónoma del Estado de México, pp. 37-73.

Flaquer, Lluís (1997), "La emancipación familiar de los jóvenes", en Revista de estudios de juventud, núm. 39, Madrid, Instituto de la Juventud, pp. 37-46.

Gallegos, M. de Jesús, Eloísa Gallegos, María Vázquez y David Sarracino (2017), "Enseñanza semipresencial como alternativas formativas del siglo XXI", en Redes académicas, docencia e investigación educativa, Lima, Red Educativa Mundial, pp. 175-181.

González, Edgar, Pablo Meira y Cynthia Martínez (2015), "Sustentabilidad y Universidad: retos, ritos y posibles rutas", en Revista de la Educación Superior, vol. 44, núm. 175, México, ANUIES, pp. 69-93.

González, Julia, Robert Wagenaar y Pablo Beneitone (2004), "Tuning-América Latina: un proyecto de las universidades", en Revista Iberoamericana de Educación, núm. 35, Madrid, Organización de Estados Iberoamericanos para la Educación, la Ciencia y la Cultura, pp. 151-164.

Guadarrama, Rocío, Alfredo Hualde y Silvia López (2016), La precariedad laboral en México. Dimensiones, dinámicas y significados, México, UAM.

Guzmán, Carlota (2017), "Las nuevas figuras estudiantiles y los múltiples sentidos de los estudios universitarios", en Revista de la Educación Superior, vol. 46, núm. 182, México, ANUIES, pp. 71-87.

Guzmán, Carlota (2004), "Los estudiantes frente a su trabajo: un análisis en torno a la construcción del sentido del trabajo", en Revista Mexicana de Investigación Educativa, vol. 9, núm. 22, México, COMIE, pp. 747-767.

Hualde, Alfredo y Arcelia Serrano (2005), "La calidad del empleo de asalariados con educación superior en Tijuana y Monterrey. Un análisis cuantitativo", en Revista Mexicana de Investigación Educativa, vol. 10, núm. 25, México, COMIE, pp. 345-374.

Johnson, Harry (1975), On economics and society. Selected essays, Chicago, University of Chicago Press.

Loyzaga, Octavio (2009), "Estabilidad: duración de las relaciones de trabajo", en Alegatos, núm. 72, México, UAM-Azcapotzalco, pp. 67-81.

Macías, Ma. Carmen (2013), "Las nuevas formas de contratación y sus repercusiones en los derechos laborales. Estudio desde el marco jurídico laboral vigente", en Revista Latinoamericana de Derecho Social, núm. 16, México, Universidad Nacional Autónoma de México, pp. 83-121.

Miller, Dinorah (2009), La equidad en la universidad: el Programa Nacional de Becas (PRONABES) y la condición de juventud de los estudiantes. Una mirada desde la UAM, México, ANUIES.

Mincer, Jacob (1974), Schooling experience and earnings, Cambridge, National Bureau Economics Research.

Mora, Minor y Orlandina De Oliveira (2009), "Los jóvenes en el inicio de la vida adulta: trayectorias, transiciones y subjetividades", en Estudios sociológicos, vol. 27, núm. 79, México, El Colegio de México, pp. 267-289.

Ramírez, Rosalba (2013), Cambiar, interrumpir o abandonar. La construcción de experiencias de los estudiantes en su tránsito por una institución de educación superior tecnológica, México, ANUIES.

Reynoso, Carlos (2017), "La precarización jurídica del trabajo", en Alegatos, vol. 29, núm. 89, México, UAMAzcapotzalco, pp. 21-36.

Rubio, Julio (2006), La política educativa y la educación superior en México. 1995-2006: un balance, México, Fondo de Cultura Económica.

Salas, Iván Alejandro y Lucio Flores (2016a), "Análisis de las condiciones laborales de los estudiantes de dos carreras de una universidad pública mexicana. Una 
aproximación basada en conjuntos de lógica difusa", en Revista Iberoamericana de Educación Superior, vol. 7, núm. 20, México, Universidad Nacional Autónoma de México-Instituto de Investigaciones sobre la Universidad y la Educación/Universia, pp. 21-41.

Salas, Iván Alejandro y Lucio Flores (2016b), "Modelado de conjuntos difusos para cuantificar la calidad del empleo. Una aproximación conceptual", en Revista Ciencia y Universidad, núm. 34, Culiacán, Universidad Autónoma de Sinaloa, pp. 7-36.

Salas, Iván Alejandro y Favio Murillo (2013), "Los profesionistas universitarios y el mercado laboral mexicano: convergencias y asimetrías", en Revista de la Educación Superior, vol. 42, núm. 165, México, ANUIES, pp. 63-81.

Singly, François (2005), "Las formas de terminar y de no terminar la juventud", en Revista de Estudios de fuventud, núm. 71, Madrid, Instituto de la Juventud, pp. 109-119.

Trow, Martin (1974), "Problems in the transition from elite to mass higher education", en Twentieth-Century Higher Education: From Elite to Mass to Universal, Baltimore, The Johns Hopkins University Press, pp. 86-142.
Universidad de Guadalajara (UDG) (2016), Informe de Actividades CUCSH 2015-2016, Guadalajara, Universidad de Guadalajara.

Valarezo, Karina y José Miguel Tuñez (2014), "Responsabilidad Social Universitaria. Apuntes para un modelo de RSU”, en Revista de Comunicación, núm. 13, Piura, Universidad de Piura, pp. 84-117.

Weiss, Eduardo (2012), "Los estudiantes como jóvenes. El proceso de subjetivación", en Perfiles Educativos, vol. 34, núm. 135, México, Universidad Nacional Autónoma de México-Instituto de Investigaciones sobre la Universidad y la Educación, pp. 134-148.

Wit, Hans (2011), "Globalización e internacionalización de la educación superior", en RUSC. Universities and Knowledge Society fournal, vol. 8, núm. 2, Barcelona, Universitat Oberta de Catalunya, pp. 77-84.

Yamasaki, Sakura y Astrid Spreitzer (2006), "Beyond methodological tenets. The world of QCA and SNA and their benefits to policy analysis", en Innovative comparative methods for policy analysis. Beyond the quantitative-qualitative divide, Nueva York, Springer, pp. 95-120. 


\section{Anexo 1. Tablas de verdad para el eje analítico asociado a la sociología de la juventud}

\begin{tabular}{|c|c|c|c|c|}
\hline \multicolumn{4}{|c|}{ Variables de entrada } & \multirow{2}{*}{$\begin{array}{c}\text { Salida } \\
\text { Etapa de la vida }\end{array}$} \\
\hline Edad & Estado Civil & Composición familiar & Destino del ingreso & \\
\hline Menor a 30 años & Soltero & Núcleo de origen & Personal & Joven \\
\hline Menor a 30 años & Soltero & Núcleo de origen & De apoyo a terceros & Joven \\
\hline Menor a 30 años & Soltero & Independiente al núcleo de origen & Personal & Joven \\
\hline Menor a 30 años & Soltero & Independiente al núcleo de origen & De apoyo a terceros & Adulto \\
\hline Menor a 30 años & Actualmente o alguna vez en pareja & Núcleo de origen & Personal & Joven \\
\hline Menor a 30 años & Actualmente o alguna vez en pareja & Núcleo de origen & De apoyo a terceros & Adulto \\
\hline Menor a 30 años & Actualmente o alguna vez en pareja & Independiente al núcleo de origen & Personal & Adulto \\
\hline Menor a 30 años & Actualmente o alguna vez en pareja & Independiente al núcleo de origen & De apoyo a terceros & Adulto \\
\hline Igual o mayor a 30 años & Soltero & Núcleo de origen & Personal & Joven \\
\hline Igual o mayor a 30 años & Soltero & Núcleo de origen & De apoyo a terceros & Adulto \\
\hline Igual o mayor a 30 años & Soltero & Independiente al núcleo de origen & Personal & Adulto \\
\hline Igual o mayor a 30 años & Soltero & Independiente al núcleo de origen & De apoyo a terceros & Adulto \\
\hline Igual o mayor a 30 años & Actualmente o alguna vez en pareja & Núcleo de origen & Personal & Adulto \\
\hline Igual o mayor a 30 años & Actualmente o alguna vez en pareja & Núcleo de origen & De apoyo a terceros & Adulto \\
\hline Igual o mayor a 30 años & Actualmente o alguna vez en pareja & Independiente al núcleo de origen & Personal & Adulto \\
\hline Igual o mayor a 30 años & Actualmente o alguna vez en pareja & Independiente al núcleo de origen & De apoyo a terceros & Adulto \\
\hline
\end{tabular}

Fuente: elaboración propia. 
Anexo 2. Tablas de verdad para el eje analítico asociado a la teoría del capital humano

\begin{tabular}{|c|c|c|c|c|c|}
\hline \multicolumn{5}{|c|}{ Variables de entrada } & \multirow{2}{*}{$\begin{array}{c}\text { Salida } \\
\begin{array}{c}\text { Nivel de } \\
\text { habilitación }\end{array}\end{array}$} \\
\hline Situación laboral & Experiencia laboral & $\begin{array}{l}\text { Empleo por } \\
\text { el tipo de } \\
\text { contratación }\end{array}$ & $\begin{array}{l}\text { Percepción de afinidad } \\
\text { carrera - empleo }\end{array}$ & $\begin{array}{c}\text { Percepción de la } \\
\text { orientación de la } \\
\text { formación }\end{array}$ & \\
\hline Desocupado & - & - & - & - & Nulo \\
\hline Ocupado & Nula & Informal & Baja & Al estudio & Bajo \\
\hline Ocupado & Nula & Informal & Baja & Al empleo & Bajo \\
\hline Ocupado & Nula & Informal & Alta & Al estudio & Bajo \\
\hline Ocupado & Nula & Informal & Alta & Al empleo & Medio \\
\hline Ocupado & Nula & Formal & Baja & Al estudio & Bajo \\
\hline Ocupado & Nula & Formal & Baja & Al empleo & Bajo \\
\hline Ocupado & Nula & Formal & Alta & Al estudio & Medio \\
\hline Ocupado & Nula & Formal & Alta & Al empleo & Medio \\
\hline Ocupado & Poca experiencia & Informal & Baja & Al estudio & Bajo \\
\hline Ocupado & Poca experiencia & Informal & Baja & Al empleo & Bajo \\
\hline Ocupado & Poca experiencia & Informal & Alta & Al estudio & Medio \\
\hline Ocupado & Poca experiencia & Informal & Alta & Al empleo & Medio \\
\hline Ocupado & Poca experiencia & Formal & Baja & Al estudio & Bajo \\
\hline Ocupado & Poca experiencia & Formal & Baja & Al empleo & Medio \\
\hline Ocupado & Poca experiencia & Formal & Alta & Al estudio & Medio \\
\hline Ocupado & Poca experiencia & Formal & Alta & Al empleo & Alto \\
\hline Ocupado & Trayectoria en desarrollo & Informal & Baja & Al estudio & Bajo \\
\hline Ocupado & Trayectoria en desarrollo & Informal & Baja & Al empleo & Medio \\
\hline Ocupado & Trayectoria en desarrollo & Informal & Alta & Al estudio & Medio \\
\hline Ocupado & Trayectoria en desarrollo & Informal & Alta & Al empleo & Alto \\
\hline Ocupado & Trayectoria en desarrollo & Formal & Baja & Al estudio & Medio \\
\hline Ocupado & Trayectoria en desarrollo & Formal & Baja & Al empleo & Medio \\
\hline
\end{tabular}




\begin{tabular}{|l|l|l|l|l|l|}
\hline Ocupado & Trayectoria en desarrollo & Formal & Alta & Al estudio & Alto \\
\hline Ocupado & Trayectoria en desarrollo & Formal & Alta & Al empleo & Alto \\
\hline Ocupado & Trayectoria en consolidación & Informal & Baja & Al estudio & Medio \\
\hline Ocupado & Trayectoria en consolidación & Informal & Baja & Al empleo & Medio \\
\hline Ocupado & Trayectoria en consolidación & Informal & Alta & Al estudio & Alto \\
\hline Ocupado & Trayectoria en consolidación & Informal & Alta & Al empleo & Alto \\
\hline Ocupado & Trayectoria en consolidación & Formal & Baja & Al estudio & Medio \\
\hline Ocupado & Trayectoria en consolidación & Formal & Baja & Al empleo & Alto \\
\hline Ocupado & Trayectoria en consolidación & Formal & Alta & Al estudio & Alto \\
\hline Ocupado & Trayectoria en consolidación & Formal & Alta & Al empleo & Alto \\
\hline
\end{tabular}

Fuente: elaboración propia.

Anexo 3. Tablas de verdad para el eje analítico asociado a la política educativa

\begin{tabular}{|c|c|c|c|}
\hline \multicolumn{3}{|c|}{ Variables de entrada } & \multirow{2}{*}{$\begin{array}{c}\text { Salida } \\
\text { Tipo de estudiante }\end{array}$} \\
\hline Dedicación al estudio & Edad & $\begin{array}{c}\text { Apoyo económico institucional } \\
\text { y/o externo }\end{array}$ & \\
\hline Exclusiva & Dentro de la cohorte de cobertura & Con apoyo & Estudiante ideal \\
\hline Exclusiva & Dentro de la cohorte de cobertura & Sin apoyo & Estudiante ideal \\
\hline Exclusiva & Fuera de la cohorte de cobertura & Con apoyo & Estudiante ideal \\
\hline Exclusiva & Fuera de la cohorte de cobertura & Sin apoyo & Estudiante ideal \\
\hline Orientada al estudio & Dentro de la cohorte de cobertura & Con apoyo & Estudiante ideal \\
\hline Orientada al estudio & Dentro de la cohorte de cobertura & Sin apoyo & Estudiante ideal \\
\hline Orientada al estudio & Fuera de la cohorte de cobertura & Con apoyo & Estudiante atípico \\
\hline Orientada al estudio & Fuera de la cohorte de cobertura & Sin apoyo & Estudiante atípico \\
\hline Orientada al trabajo & Dentro de la cohorte de cobertura & Con apoyo & Estudiante atípico \\
\hline Orientada al trabajo & Dentro de la cohorte de cobertura & Sin aроуо & Estudiante atípico \\
\hline Orientada al trabajo & Fuera de la cohorte de cobertura & Con apoyo & Estudiante atípico \\
\hline Orientada al trabajo & Fuera de la cohorte de cobertura & Sin apoyo & Estudiante atípico \\
\hline
\end{tabular}

Fuente: elaboración propia. 
Anexo 4. Tablas de verdad para la tipología de estudiantes de acuerdo a la relación educación superior-empleo

\begin{tabular}{|c|c|c|c|}
\hline \multicolumn{3}{|c|}{ Variables de entrada } & \multirow{2}{*}{$\begin{array}{c}\text { Salida } \\
\text { Tipo de estudiante }\end{array}$} \\
\hline Sociología de la juventud & Teoría del capital humano & Política educativa & \\
\hline Joven & Nulo & Estudiante ideal & Estudiante \\
\hline Joven & Nulo & Estudiante atípico & Estudiante \\
\hline Joven & Bajo & Estudiante ideal & Estudiante que trabaja \\
\hline Joven & Bajo & Estudiante atípico & Estudiante que trabaja \\
\hline Joven & Medio & Estudiante ideal & Estudiante que trabaja \\
\hline Joven & Medio & Estudiante atípico & Transición \\
\hline Joven & Alto & Estudiante ideal & Transición \\
\hline Joven & Alto & Estudiante atípico & Trabajador que estudia \\
\hline Adulto & Nulo & Estudiante ideal & Estudiante \\
\hline Adulto & Nulo & Estudiante atípico & Estudiante \\
\hline Adulto & Bajo & Estudiante ideal & Transición \\
\hline Adulto & Bajo & Estudiante atípico & Transición \\
\hline Adulto & Medio & Estudiante ideal & Trabajador que estudia \\
\hline Adulto & Medio & Estudiante atípico & Trabajador que estudia \\
\hline Adulto & Alto & Estudiante ideal & Trabajador que estudia \\
\hline Adulto & Alto & Estudiante atípico & Trabajador que estudia \\
\hline
\end{tabular}

Fuente: elaboración propia.

\section{Cómo citar este artículo:}

Arellano-Martínez, Jorge-Alberto e Iván-Alejandro Salas-Durazo (2019), “¿Puntos de partida y llegada simétricos? Una tipología basada en la relación educación-empleo", en Revista Iberoamericana de Educación Superior (RIES), México, UNAM-IISUE/Universia, vol. x, Núm. 28, pp. 3-24, DOI: https://doi.org/10.22201/iisue.20072872e.2019.28.426 [consulta: fecha de última consulta]. 